\title{
Foresighting organizational learning equilibrium in European universities
}

\author{
Stig A. Selmer-Anderssen ${ }^{1}$ • Jan E. Karlsen ${ }^{2}$ (D) \\ Received: 31 August 2016 / Accepted: 24 November 2016/Published online: 9 December 2016 \\ (C) The Author(s) 2016. This article is published with open access at Springerlink.com
}

\begin{abstract}
This article presents a short journey into the realm of foresighting exploration and exploitation of knowledge. Universities aim to achieve a prolific balance between research, education and third mission activities. Presently, the rate of change (and especially exogenous change) have reached a level that has given researchers and managers alike reason to reflect upon and be concerned about whether this balance could or should be maintained in the future. The increase in levels of change will necessarily require a momentum of organizational learning within the university. This applies whether the aim is to maintain the balance or to shift the balance in a well-planned manner. In this article, we use a classical organizational learning model to envision and simulate how a primary driver (mutual learning) in the balance between exploration and exploitation of knowledge in universities may evolve over time. Using 2010 as baseline and the European Higher Education Area (EHEA) as contextual development path, the simulation process highlights parameters of organizational performance and competitive advantages
\end{abstract}

Authors comments The article originates from observations and assessments of European university adaptations over two decades, and from Agent Based Model simulation workshops and foresight exercises highlighting decision making and learning processes of prototypical universities in a foresight management framework.

Stig A. Selmer-Anderssen

stig.selmer-anderssen@uis.no

Jan E. Karlsen

jan.e.karlsen@uis.no

1 Quality Assurance Department, University of Stavanger, 4036 Stavanger, Norway

2 Faculty of Social Sciences, University of Stavanger, 4036 Stavanger, Norway such as learning rates, turnover and environmental turbulence stepwise at year 2015, 2020, 2025 and 2030. Prototypical European universities are contrasted with hypothetical organizations exposed to even more vibrant environments.

Keywords EHEA · Universities · Organizational learning · Organized anarchies $\cdot$ Simulated futures

\section{Introduction}

Consider organized anarchies and organizational learning in European universities. It is all about organizational ambidexterity [1-3] and punctuated equilibrium [4]. It is at the very core of universities' modus operandi to have one arm working at the optimal dissemination of the best of our present knowledge while the other works equally hard on replacing this knowledge with something even better.

During several decades, higher education institutions all over the world have been the target of reforms seeking to overcome outdated university practices and governance [5]. Higher education institutions and systems in Europe have experienced a generous share of these initiatives. A number of educational sector and public sector reforms were launched during the latter part of the last century. Since the turn of the century, the rate of change in the European higher education sector has increased dramatically [6, p. 759]. Important factors behind this were the Bologna Declaration in 1999 and the Lisbon Strategy in 2000, aimed at European integration and at making Europe the world's most competitive and dynamic knowledge-based economy by 2010. The rate of change (and especially exogenous change) has reached a level that could give reason to reflect upon and be concerned about whether the institutional balance could - or should - be maintained in the future. 
Organizational research has provided us with numerous tools and models to assist us in making such reflections as part of our daily work. However, March's [7] highly influential and widely used exploration-exploitation (E-E learning) model would seem highly appropriate for such purposes. March's model has spurred a plethora of papers presenting extensions, reconceptualization and uses of the exploration-exploitation model, including a Special Research Forum on the model in Academy of Management Journal [8]. A recent review paper [9] found that 145 papers based on the model were published in the period 1992-2012. Nevertheless, a search for applications of the model to higher education institutions showed only a few, and none that specifically addressed the particular organizational form used by most higher education institutions.

This article reports the quest to apply the E-E learning model to higher education institutions in Europe, and to use the model to generate simulations of prototypical institutions within the European higher education area (EHEA) in the period 2010 to 2030 .

\section{The challenge}

In March's seminal article Exploration and exploitation in organizational learning [7], March examines problems regarding the balancing of resources allocated to the exploration of new possibilities with those allocated to exploitation of what is already known. With his model as our outset, we wanted to learn whether (and eventually how) the balance between exploration and exploitation in organizational learning could be used as a foresighting tool. The aim was to reflect upon and possibly gain insight about how universities in Europe might expect their mutual learning to develop under different conditions. The borders of the EHEA was seen as the natural limits for this foresighting endeavour.

Thus, we saw this task as a challenge consisting of two pieces:

1. Would application of March's E-E learning model be viable and applicable to higher education institutions, and for the foresighting purpose?

- We thought of this as the explorative and methodological part of our effort.

2. If appropriate, could the model be applied as a tool to envision possible future developments for prototypical higher educational institutions in Europe?

- We thought of this as the contextual and exploitative part of the challenge.
March's E-E model hinges on mutual learning within the organization to achieve the necessary balance. The term $m u$ tual learning refers to how the organization learn from its members while at the same time its members learn from the organization. The three basic elements of the model are the external reality (the organization's environment; the market), the organizational code (the organization culture, the conventional wisdom within the organization), and the organization's members. How the balance develops over time will therefore depend on the individual learning rate of the members and the learning rate of the organization as well as factors like turnover and environmental turbulence. The E-E model is presented somewhat more in detail in the next section.

The E-E model has been repeatedly shown to apply well to companies in numerous sectors [9, 10], for companies supporting the common organizational form of having a single formal superior authority within the corporation/organization. Usually this is implemented by having a board or similar construct acting as the single superior authority. However, we were concerned that the E-E model might contain elements that presupposed this organizational form. If so, the model might not apply equally well to European higher education institutions as these have more than one formal superior authority. In most European countries, they have three formal (de jure) authorities [11, 12]; academic freedom, ministry governance and institutional autonomy.

The first effort would be to clarify whether the E-E model contained any elements as mentioned above. This includes seeking out possibilities for adapting the model to higher education institutions and is reported in section "The Models". March [7] examined the E-E model by simulating how the mutual learning affected the balance under different conditions. The second effort includes performing corresponding simulations for a prototypical higher education institution within the EHEA. This is reported in section "The context and the simulations".

We see universities as "prototypical" when they are governed by the three formal authorities, are engaged in research, education and third mission activities, and simultaneously cater to exploration and exploitation of knowledge.

\section{The models}

In clarifying how the E-E model could be applied to universities, it is necessary to present three models:

- The E-E learning model itself;

- The superior group (SG) choice model that is implemented as an integral part of the E-E learning model;

- The garbage can model (GCM) of organizational choice. 


\section{The E-E learning model}

Since the publication of the E-E model, the balance between exploration and exploitation has seen a dramatic increase in use within organizational analysis as well as in organizational design, organizational learning, and even organizational survival. While the general concept is seen to dominate the field [13], the model of balancing exploration and exploitation was presented by March [7] as a way to enable organizational ambidexterity in an organization. Such ambidexterity relies on finding a balance that lets the organization be creative and adaptive while at the same time relying successfully on its traditional and proven business methods. Companies that focus only on exploration expose themselves to bearing "the cost of experimentation without gaining many of its benefits" [7, p. 71]. Companies that focus only on exploitation "are likely to find themselves trapped in suboptimal stable equilibria" [7, p. 71].

The E-E model envisions the interplay between the organization's beliefs and the personal beliefs of each individual in the organization. The core of the model is how organizational and individual beliefs contribute to each other through a process of mutual learning. The model relates organizational and individual beliefs to an external reality. The external reality is assumedly known, and it is independent of organizational and individual beliefs. This interaction between individuals, organization and the external reality depicts a central mechanism in the balance between exploration and exploitation of knowledge in organizations [13] in a parsimonious and very elegant way. The model encompasses a small number of core elements, designed and interconnected to contain substantial learning phenomena [7].

At its core, we find these items:

- An external reality in $m$ dimensions. The dimensions are independent of any beliefs about them, and independent of each other.

- An organizational code in $m$ dimensions. This code depicts the reality as seen by the organization. At the very outset of an organization, the organizational code will be empty.

- A population of $n$ individuals within the organization. Each individual has an individual code in $m$ dimensions, depicting each individual organization member's beliefs. The individuals may carry along beliefs as they become members of the organization.

- The organizational learning rate; how effectively the organization code learns from its members.

- The socialization rate of the individuals; how effectively the individuals learn from the organizational code.

- The rate of turbulence in the external environment.

- The rate of internal turnover, i.e. changes of individual beliefs.
The model do not presuppose any agreement between the organizational code and the external reality. On the contrary, at the outset of an organization, the organizational code differs from the external reality at each and every of the dimensions. Thus, how well the organizational code reflects the actual reality at any given point is a matter of the choices made in the organization. In the E-E model, the choice mechanism for code learning is the 'superior group' model.

As discussed below, the superior group model presupposes a single superior authority. All universities in Europe have more than one superior authority. We argue that choice models that do not presuppose a single superior authority might be more apt when applying the E-E model to universities. In particular, we propose that the choice model presented in the seminal article by Cohen et al. "A Garbage Can Model of Organizational Choice" [14] may be better suited than the superior group choice model.

\section{The superior group choice model}

At the very outset of an organization, the organizational code of the E-E model do not contain any beliefs at all. Over time, the organizational code builds up, according to its learning rate and its model for how to learn, as implemented through the superior group model.

The E-E model is a dynamic model, depicting the evolution of organizations over time. Time is divided into periods following each other. It is in the transitions from one period to the next that the state of affairs is sampled, and the books updated. The organizational code is not dictated by the external reality, and at each transition point the organization is free to choose any value it wants on any dimension of the organizational code. With the superior group choice model, the values are chosen in this way:

- The superior group consists of all members who have an individual code that reflects reality better than the present organizational code.

- If the value of the organizational code on a particular dimension agrees with the value a majority of the members of the superior group hold at that dimension, the value for that dimension of the organizational code is kept unchanged.

- If a majority of the superior group differs from the value of the organization code on a particular dimension, it might be changed or it might be kept unchanged. The probability that it is kept unchanged increases with lower organizational learning rate, and with increasing rate of dissent within the superior group.

Thus, the superior group is not an organizational entity with a fixed membership, and the membership might or might not change from one period to the next. At the outset, the superior 
group will consist of all members of the organization (or 'all minus one ${ }^{1}$ ). When there is no internal or external turbulence, the organizational code converges on reality, with the speed of convergence depending on the learning rates of the organization and its members. However, there is a risk that subsets of dimensions form stable rings, where the values of the organizational code and the codes of the members of the superior group are shared but differ from the reality, or where the values oscillate between having no value or the wrong value. This makes convergence to reality impossible for the affected dimensions. The result will be stable states where the organizational code reflects reality only to a certain fixed degree. March [7] refers to this as the knowledge equilibrium, and shows that this lock-in to organizational codes that poorly reflects reality can be better avoided when the organization have high learning rate while the members have low learning rates.

From a democratic outset where all members have an equal say in the forming of the organizational code, the superior group process proceeds by continuously excluding members that cannot boast an individual code that reflects reality better than the organization's present code and including those who can. In a turbulence-free environment, this will lead to an increasingly narrower knowledge elite being involved in the changing of organizational code, until the organizational code finally mimics reality as good as it can by reaching its equilibrium state. However, turbulence happens, and both external turbulence and internal turbulence (turnover) directly affects which members of the organization will be included in the superior group. Turbulence thereby counteracts the tendency of the superior group model to form a knowledge elite.

In the superior group model, only a subgroup gets to have a say in what values the institutional code should contain. This group is not open to everybody. Only individuals fulfilling certain criteria is included in the group. The superior group model is a single authority model.

\section{The garbage can choice model}

Universities in Europe all have more than a single authority. Within the EHEA the academic freedom is one of these, also given de jure support in most participating countries. In addition, most countries have implement a de jure institutional authority as well as a de jure national authority. Organizations where choices and decisions are made under influence of multiple authorities will often have the organizational form termed organized anarchies [14] having these characteristics:

- They support multiple and divergent goals, resulting in problematic preferences

\footnotetext{
${ }^{1}$ The 'one' being the odd fellow that supports no beliefs at any dimension of reality whatsoever.
}

- They operate with unclear and usually diversified decision processes and technologies

- They experience fluent process participation. When many choice arenas are available, members cannot mostly due to time and resources restrictions attend to all choice arenas they have access to. Members then prioritize, often according to practical considerations, individual preferences or organizational needs.

Choice or decision processes having these characteristics are perceived as problematic in most (single authority) organizations. From a governance point of view, they can be perceived as problematic also for universities supporting multiple authorities (see e.g. [15]). From an institutional or sectorial perspective however, the form of organized anarchies may improve the resilience of universities [11].

Cohen et al. [14] devised a model for choice and decision processes in organized anarchies. This model can be used also for multi authority organizations [11]. Using the E-E model with a garbage can choice model rather than a superior group choice model would enable use of the E-E model also in the context of universities.

The garbage can model evaluates different ways the members may take part in the different choices, different ways decisions can be made, and different ways the energy is distributed among the members. This energy is needed to solve problems. Members attend to choice opportunities in which problems may be solved [8]. In each such choice opportunity, decisions may be made in three different ways:

- By resolution: The available energy enables a choice to be made, which also actually resolves a problem.

- By oversight: A choice opportunity where there is no problem attached. The available energy may then be used to make a choice quickly and with a minimum of energy.

- By flight: If a choice opportunity do not have sufficient energy to resolve the problem(s) present, a problem may be transferred to another arena. In the present choice arena it may then be possible to make a choice, as the energy required to resolve the transferred problem(s) is no longer a constraint.

The E-E model divides time into periods following each other, with choices being made regarding the values of the organization code in the transitions from one period to the next. With the garbage can choice model integrated into the E-E model, the values are chosen in this way:

- All individuals participating in a choice bring their individual code to the choice opportunity.

- All individuals cannot attend to all choice opportunities. The participation factor indicates the probability that an individual will attend to any particular choice opportunity. 
- If a choice is made by resolution, the value of the particular dimension of the organization code is chosen to be the value held by a majority of the participating individuals for that particular dimension.

- If a choice is made by flight, this particular choice opportunity pushes the problem of evaluating the value of the dimension of the organization code onto other choice arenas. The value of that particular dimension is therefore left unchanged in the organization code.

- If a choice is made by oversight, the choice opportunity do not view that dimension of the code as one of particular interest for the institution, and it defaults to the value of the corresponding dimension of reality.

\section{The context and the simulations}

The three models (E-E, SG and GCM, respectively) briefly described above are integrated as the basis for the simulations performed and described below. By implementing the garbage can choice mechanism in the E-E model, the model will be applicable to prototypical European universities within a context of the EHEA. These are multi authority organizations supporting academic freedom as well as ministry governance and institutional autonomy, and as such a perfect fit for a combination of the superior group choice model and the garbage can choice model in their organizational learning efforts.

\section{The EHEA as context}

European higher education institutions have experienced numerous reforms and changes through the educational sector and public sector reforms that were launched before of the turn of the century. Since then, however, the rate of change has nevertheless increased dramatically [6, p. 759]. Central factors behind this was the Bologna Declaration in 1999 and the Lisbon Strategy in 2000, with aims to integrate the higher education across all of Europe and govern the sector towards common goals being collectively referred to as 'Europe 2020' [16:3].

The rate of change instigated by the Europe 2020 processes is characterised as unprecedented in the higher education world [17: p. xiii]. The processes has been described as "fundamental", "major" and "profound", and the effects has been described as "remarkable" and a "dramatic restructuring of higher education" [18]. The European Commission developed the Open Method of Coordination (OMC) as a tool to coordinate and govern the huge amount of changes that would have to be made to the national educational systems in Europe. The OMC was designed as a tool for mutual policy learning and knowledge exchange between the participation nations [17]. It turned out to be very efficient, even 'revolutionary' [19].

The fundamental goal of establishing the higher education institutions across all of Europe in a single European Higher Education Area (EHEA) was achieved in 2010, as the Bucharest-Vienna Ministerial Conference declared the establishment of the European Higher Education Area [20]. However, this was a goal achieved with the narrowest possible margin. At the time of declaration of the EHEA, the most fundamental elements were in place across most participating nations. For the institutions and many of the national higher education systems, the declaration of the EHEA was when the real work started [21]. The member countries were at quite different maturity levels at the outset in 2010, and the communique from the EHEA Ministerial Conference in 2015 underscores that the diversity across nation has not decreased sufficiently. At the Ministerial Conference a new version of the European Standards and Guidelines for Quality Assurance in the European Higher Education Area (ESG) was adopted [22]. The Standards and Guidelines (and especially the new 2015 edition) have required most member countries to make changes to their national legislation. These changes are at different stages in the different member countries. The Ministerial Conference outlines the major challenges that are to be met in the following years in their conference communiqués. The present communique and the strategies for the EHEA and its different workgroups are good indications of the rate of change in the subsequent years [23: 1-2].

The Ministerial Conference now aims to tighten the grip on member countries that show a relaxed attitude towards the criteria, stating that EHEA will be «addressing the issue of non-implementation of key commitments in time for our next meeting» [23: 3].

\section{Challenges when using the EHEA as simulation arena}

For the purposes of applying the E-E model to higher education institutions, the EHEA as described above will for several reasons not be the simplest possible context for our simulation modelling.

The special research forum on the E-E model being reported in the Academy of Management Journal [8] posits the relation between ambidexterity and punctuated equilibrium as one of the fundamental issues in using the E-E model. While the very mission of universities necessitates applying both exploration and exploitation to research as well as to education and third mission in an ambidextrous way, the risks of having to adapt to punctuated equilibria still applies. The empirical test of punctuated equilibria in organizational transformation of companies performed by Romanelli and Tushman [24] shows that this risk is real. Major shifts in the regulatory environment has been found to serve as punctuated equilibria [25]. Thus the context of the EHEA should be 
analysed for possible sources for punctuations of the equilibria.

The EHEA presents us with a rich set of information on the evolution as well as for previous plans for the future (see http://www.ehea.info/). In summary, these show that the environment have not achieved the stability that is required for using the E-E model as a stationary model. The present strategic plans for development of the EHEA shows that such stabilisation of the rate of change is not achieved by 2015 . However, the present plans encompass all nations having implemented all required elements by the year 2020 [23], indicating that stability will be reached in 2020 .

- The 2010 "Budapest-Vienna Declaration on the European Higher Education Area" [20] signed by 47 nations instigated the EHEA and posited an increasing rate of change across the policy areas of the EHEA.

- The document «The European Higher Education Area in 2012» implementation report [26] presented to the ministerial conference in Bucharest in 2012, as well as the communiqué from the ministerial meeting [27], showed that the increase posited in 2010 would have to be maintained to meet goals set for 2015.

- The document «The European Higher Education Area in 2015» implementation report [28] presented to the ministerial conference in Yerevan in 2015 stated that «The Bologna process has arrived at a crucial point» [28, p. 3]. Nevertheless, the communiqué from the ministerial meeting stated that «By 2020 we are determined to achieve an EHEA where our common goals are implemented in all member countries» [23, p. 1-2]. A lot of work is still remaining, in institutions as well as in national higher education systems. Also, in many cases the changes in the institutions is done in response to changes in the national systems, therefore often lagging the system changes by one to three years.

- The EHEA documents do not yet indicate any new changes to be introduced in EHEA after 2020.

Based on these documents, the evolution of the EHEA and its national systems and higher education institutions might see this evolution in the period 2010-2030:

- The period 2010-2015 will see a steady increase in rate of change.

- Starting in 2015, this increase will level off, and remain at the same rate until 2020.

- While the changes per plans should be completed by 2020, the experience from the Bologna process up to now indicates that this goal might not be fully met, so changes will still remain. Institutions will lag system changes in many cases. For these reasons, the rate of change could be expected to continue, but gradually decrease. The expectation used in the simulations is that the annual decrease will be lower than the annual increase in the period 2010-2015, and that the process will in 2030 arrive at the same rate of change as in 2010 .

- There will be a steady increase in internationalisation and staff mobility from 2010 to 2020, and a further increase as the increase expands to administrative staff as well from 2015. As no further plans exist, the level may stay at a steady level from 2020 to 2025 , and then decrease from 2025 to 2030.

Based on these presumptions, our simulations will punctuate the change equilibrium at least at two points; from increased rate of change to stable rate of change in 2015 , and from stable rate of change to decreasing rate of change in 2020. The simulations will thus start at the birth of EHEA in 2010 , and will run to 2030 .

The motivations and current strategies for the EHEA include giving high attention to increased internationalisation (of study programmes as well as their environments for delivery) and increased level of mobility (of students as well as scientific and administrative staff). The intended results include better harmonisation between European higher education institutions as well better harmonisation between the national higher education systems. The effect of this, transferred to our model scenario, will be changes in the endogenous reality of the organization, seen as changes to individual dimensions of the member's belief. As no targets are set for the EHEA regarding this, the simulations are done under the assumptions that this factor will be increasing in a slow but steady way throughout the simulation period.

In addition, the EHEA as simulation scenario poses a few minor challenges when doing simulations based on the E-E model. As per March's 1991 article, the E-E model hinges on a stationary model of mutual learning. The term stationary indicates that the parameters governing the transitions from one period to the next are kept unchanged (stationary) throughout the simulations. But the EHEA scenario described above requires not only that the transition parameters change from one period to the next, but that the rate of change may be changed as well (at the points in time where the equilibrium is punctuated). To allow for this, non-stationary (dynamic) versions of the models were used in the simulations.

\section{The E-E model simulations}

The simulations were done using, as far as possible, identical scenarios and variable settings as those used in March [7] article for the E-E model and in Cohen et al. [8] article for the garbage can choice model. In summary, these are as follows:

- Number of periods in the E-E model: 80 
- Number of members in the organization: 50

- Number of dimensions in reality/organizational code/individual code: 30

- Number of iterations of each simulation scenario: 80

- Number of choice opportunities in each period (for the garbage can choice model): 50

- Number of iterations of each choice opportunity: 80

\section{The results for the EHEA scenarios}

In Table 1, the results from the simulations show how well the organizational code reflects the external reality of the EHEA during the period. The period used for simulations are 20102030 , but since all 2010 variables must start at 0 , they are suppressed. The table results give the percentage of the external reality that is reflected in the organization's code.

The figures used to calculate the percentages are the number of dimensions of the organization code that correspond to the respective dimension of the external reality. We repeated the simulations 80 times for each of the 80 periods involved, as in the original E-E model [7]. Then we recorded the results. The percentage shows the average result across the simulations. In the scenarios involving the garbage can model, each of these simulations contain 50 choice arenas within each period. For each of these, 80 repetitions are performed. In this table, the results across the two models are shown for each of three adaptation levels.

One notable feature of these results are the very similar results achieved for the three different GCM adaptations for all the time periods. While achieving low level of code knowledge in 2015, the three series end up at high levels of code knowledge in 2030, independent of adaptation rate.

Another feature is that the SG having low adaptation rate show a stable code knowledge throughout all time periods, while when having medium and high adaptation rate experience a certain increase from 2015 to 2030 .

As contrasting scenarios, we also simulated foresighting code knowledge dynamics in cases that are quite different from the EHEA contextual scene, as shown in Table 2. The system dynamics used is one with evolution over time

Table 1 Foresighting code knowledge in EHEA 2010-2030

\begin{tabular}{lcccc}
\hline Adaptation model \& rate & 2015 & 2020 & 2025 & 2030 \\
\hline Garbage can, low adaptation rate & $34 \%$ & $65 \%$ & $79 \%$ & $86 \%$ \\
Superior group, low adaptation rate & $58 \%$ & $55 \%$ & $56 \%$ & $58 \%$ \\
Garbage can, medium adaptation rate & $35 \%$ & $66 \%$ & $77 \%$ & $87 \%$ \\
Superior group, medium adaptation rate & $67 \%$ & $65 \%$ & $70 \%$ & $74 \%$ \\
Garbage can, high adaptation rate & $34 \%$ & $67 \%$ & $79 \%$ & $87 \%$ \\
Superior group, high adaptation rate & $69 \%$ & $70 \%$ & $71 \%$ & $78 \%$ \\
\hline
\end{tabular}

Table 2 Foresighting code knowledge in alternative scenarios not similar to EHEA

\begin{tabular}{lllll}
\hline Adapation model \& system dynamics & 2015 & 2020 & 2025 & 2030 \\
\hline Garbage can, Low-High & $27 \%$ & $67 \%$ & $53 \%$ & $47 \%$ \\
Superior group, Low-High & $74 \%$ & $68 \%$ & $46 \%$ & $24 \%$ \\
Garbage can, High-Low & $27 \%$ & $54 \%$ & $42 \%$ & $47 \%$ \\
Superior group, High-Low & $72 \%$ & $64 \%$ & $40 \%$ & $33 \%$ \\
\hline
\end{tabular}

experiencing continuously increasing internal and external turbulence throughout the period, from low to high.

Table 2 shows possible code knowledge evolutions in other (hypothetical) organizations than the prototypical EHEA higher education institution. The simulation period is 2010 2030 as in Table 1, and again the numbers for 2010 are suppressed. The table results give the percentage of the external reality that is reflected in the organization's code.

Evolutions are shown for organizations where socialization rate and organizational learning rate are continuously increasing (indicated by 'Low-High' system dynamics) as well as continuously decreasing (indicated by 'High-Low' system dynamics) for each of the two adaptation models.

It is a notable feature of these results that for both system dynamics, the SG model show a peak code knowledge in 2015 followed by a steady decline towards 2030. Both GCM models show a peak in 2020, followed by an uneven further development up to 2030 , created by the difference in system dynamics.

\section{Discussion}

In our societies, universities are the archetypical knowledge organizations. Universities have to explore new knowledge and replace the best of our knowledge with something even better, and simultaneously exploit existing knowledge through the best possible dissemination of this present knowledge to students and the public. It is therefore paramount that the universities' culture and organizational codes continuously adapt to the external reality that the institution resides within, even in times when that external reality changes.

Adapting to changing conditions is always difficult, even for universities. According to the E-E model, the balancing act between successful exploration and successful exploitation requires that the balance between the organization's learning culture and the cultural learning in the organization can be maintained and developed. If not, the organizational code may lose its grip on reality.

In the E-E model, it is the level of correspondence between the organization code and the external reality that indicates the grip the organization has on reality. The model includes four central parameters (five in the version where the GCM choice 
model is added). These can be attuned to simulate numerous possible developments for different scenarios. Running simulations for numerous different scenarios show that many combinations and development result in maintaining or improving the knowledge level of the organization code, while other indicate more worrisome developments.

\section{Foresighting organizational adaptation processes}

The SG adaptation process and the GCM adaptation process do lead to quite different results in the evolution of organizational code knowledge. One striking difference is that the GCM adaptation process seldom suffer from the same deteriorating effects over time as seen in many scenarios that utilize SG in their organizational learning. The reason for this difference lies in the fact that the superior group may internally agree on beliefs that disagrees with reality, but the organization may still adopt these beliefs into the organization code. The GCM adaptation process will in most cases involve a larger number of individuals in the organization. The process typically include lots of arenas where some of the members meet. They exchange their beliefs, possibly with harmonizing effects, and the organization code will reflect the beliefs of a much higher percentage of the members. The SG process progresses quickly to the equilibrium level, but may not respond efficiently to changes in the external environment. The GCM process adapts slower, but will in many cases steadily move towards a higher level of code knowledge.

The learning rates of an organization generally has considerable impact on the evolution both inside organizations utilizing SG adaptation processes as well as in organizations using GCM adaptation processes. However, the results as shown in Table 1 indicates that for prototypical institutions in the specific context of the EHEA with the evolutionary parameters as assumed for the period 2010-2030, the process is quite indifferent to variations in the organizational learning rate for institutions where GCM dominates the organization's learning. As indicated in Table 2, this is not a general artefact of the model. This is instead a result of the planned evolution of EHEA as implemented in the parameters of this particular set of simulations. A consequence for the institutional strategic foresighting could be that as long as GCM dominates the organization's learning it will not be necessary to accommodate for any adjustment of the learning rate: Slow-learning institutions might experience an improvement in code knowledge being almost identical to the one experienced by quicklearning organizations.

The organizational code knowledge level achieved by the SG adaptation as shown in Table 1 illustrates some characteristic features of this adaptation type. The tendency to slightly decrease between 2015 and 2020 is due to the deterioration of code knowledge inherent in the SG process. The higher the learning rate, the more deterioration, described by March as the degenerate property under conditions of exogenous turbulence $[7, p .80]$. This result is counteracted by experiencing increased learning rates over time. In our simulations, that part of the process outweighs the degeneration from 2020 onwards.

While the initial learning rate accomplished by the GCM process is noticeably lower than for the SG process, it will over time achieve better code knowledge. This resonates with March's [7, p. 86] comment that the returns of fast learning are not all positive.

For ministries, caring about the evolution of the higher education sector on national scale more than about the evolution of each individual institution, it might be compelling to view the $S G$ as being populated not by the individuals possessing superior knowledge but by individuals possessing superior power. From such a perspective, the superior group could be populated by the organization's board and senior management team. Although it is easy to envision this as the "wet dream" of politicians, bureaucrats and NPM-proponents alike, it fails to meet the premise of the organizational code being a result of the cultural process of organizational learning. In real life the organization code reflects what we actually do believe, rather than what the people in power think we should believe.

March [7] indicates that when the organizational learning rate is high while internal turbulence (turnover) is low, the SG process favours getting to the results quickly, at the expense of the quality of the knowledge in the code. A general feature of the GCM as indicated by the simulations is that the GCM process favours getting a high quality of the knowledge in the code, at the expense of not achieving the results quickly. Relating these characterisations to the exploitation/ exploration balance, the SG adaptation would seem to be of a more exploitative nature, while the GCM would seem to be of a more explorative flair. A primary goal of strategic foresighting using models and simulations is to give suggestions on how one may proceed. By including GCM adaptation in the E-E model, higher education institutions may utilize the E-E model to give suggestions on how to proceed. This applies if the goal is to attune the organizational learning to the process of developing a more exploitative organizational culture, but equally well how to proceed if the goal is to attune the organizational learning to a process of developing a more explorative organizational culture. This would not be constrained to universities within the EHEA, but could be of utility in any organization.

\section{Conclusion and outlook}

Universities within EHEA are multi authority institutions, most of them using the organizational form of organized anarchies for many of their core activities. Our simulations indicate that this may limit the risk exposure regarding organizational 
learning and adaptation. From its inception in 2010, the EHEA has undergone an exploration phase during its first five years, experiencing search, variation, innovation and development. The 2015 EHEA Ministerial Conference clearly stated that this phase should be completed by 2020 , opening up for a phase more characterised by harvesting the results of the preceding explorative phase. At the EHEA level, the year 2020 will mark the transition from exploration to exploitation. The balancing between the two is achieved by temporal phases. This resonates well with the predominant view among researchers that the balance should be kept as a punctuated equilibrium, rather than being anagenetically ambidextrous [13, p. 694].

In the context used here, the ambidexterity question is double sided. On the one hand, ambidexterity and the chores of balancing between opposing organizational learning dynamics over time is what universities face for any planning period, short or long. On the other hand, the higher education institutions have to balance the influence from their sectorial governors (ministries) up against their institutional autonomy as well as the academic freedom.

Operating within these frames, each institution should be able to adapt to the punctuated equilibria as they occur in the EHEA. The universities need to maintain organizational learning processes that can easily adapt to and keep in phase with punctuated equilibria in their external reality. The organizational learning would need to underpin the actual changes that must take place within the organization, as the external reality moves between shifting phases of exploration and phases of exploitation, as exemplified by foresighting from 2010 to 2030 shown in Tables 1 and 2.

These simulation results do not provide reasons for concern for institutions utilizing GCM adaptation processes for organizational learning as their dominant model in the future. The simulations show that organizational learning through GCM processes are highly resilient in higher education institutions. The simulations foresee the ability to accommodate for (and actually thrive on) external as well as internal turbulence. The GCM as organizational model for universities is seemingly so robust that it in most situations will not degenerate even under conditions of external and internal turbulence. This robustness can be found also in the SG model, but to a lesser extent. The inherent degeneracy of the SG model under external turbulence is counteracted by insuring sufficient internal turnover [7, p.80]. Within the assumed evolution of EHEA as a context, we have found no institutional scenarios that result in the loss of knowledge identified by March [7] as an inherent risk. This result resonates well with former Norwegian Minister of Education Gudmund Hernes' characterization of universities as being 'super-resilient organizations' [29].

The E-E model, being a highly simplified and quite abstract model, would seem more suited as a forecasting tool than as tool for specific planning purposes. Box's [30] famous dictum that while all models are wrong, some are still useful, certainly applies. Empirical studies have shown that the E-E model has wide utility [9, 24], and we have shown that by including the GCM in the model, it will apply also to higher education institutions. In applying the E-E model to specific institutions rather than prototypical universities, this addition make it possible to evaluate for each dimension in the organizational code whether the dimension learns primarily through a SG process or primarily through a GCM process.

Universities relying dominantly on the SG adaptation process may have to watch out for signs of losing some of their grip on reality, according to our simulation results. However, they could be aided somewhat by the expected change of mode of the EHEA in 2020 due to moving from a phase of exploration and into a phase of exploitation. The simulation results still indicate that knowledge level degeneration could be the result if the institution do not take sufficient part in the internationalization or mobility activities within the EHEA, or explore other means of keeping their endogenous change rate sufficiently high.

Looking outside the realm of higher education, we suspect that an E-E model supporting superior group processes as well as garbage can processes would be of value in any organization attempting to nudge the organizational culture towards becoming more explorative, or towards becoming more exploitative.

Open Access This article is distributed under the terms of the Creative Commons Attribution 4.0 International License (http:// creativecommons.org/licenses/by/4.0/), which permits unrestricted use, distribution, and reproduction in any medium, provided you give appropriate credit to the original author(s) and the source, provide a link to the Creative Commons license, and indicate if changes were made.

\section{References}

1. Tushman ML, O'Reilly CA (1996) The ambidextrous organizations: managing evolutionary and revolutionary change. Calif Manag Rev 38(4):8-30

2. O'Reilly CA, Tushman ML (2004) The ambidextrous organization. Harv Bus Rev 82(4):74-81

3. Organization Science (2009) Special issue: Organizational Ambidexterity 20(4): 685-828

4. Tushman ML, Romanelli E (1985) Organizational evolution: a metamorphosis model of convergence and reorientation. In: Cummings LL, Staw BM (eds) Research in organizational behavior 7. JAI Press, Greenwich, pp 171-222

5. Musselin C, Teixeira PN (2014) Introduction. In: Musselin C, Teixeira PN (eds) Reforming higher education. Public policy design and implementation. Springer, Dordrecht, pp 1-17

6. Maassen P, Stensaker B (2011) The knowledge triangle, European higher education policy logics and policy implications. High Educ 61:757-769

7. March JG (1991) Exploration and exploitation in organizational learning. Organ Sci 2(1):71-78

8. Special Research Forum on the Interplay between Exploration and Exploitation. Academy of Management Journal, 49(4): 693-835 
9. Almahendra R, Ambos B (2015) Exploration and exploitation: a 20-year review of evolution and reconceptualization. Int J Innov Manag 19:1-31. doi:10.1142/S1363919615500085

10. Carrol TN (2012) Designing organizations for exploration and exploitation. J Organ Des 1(2):64-68

11. Selmer-Anderssen SA (2013) Robustness in organized anarchies. In: Karlsen JE, Pritchard RMO (eds) Resilient universities: confronting changes in a challenging world. Peter Lang, Bern, pp 149-189

12. Huisman J (2009) Coming to terms with governance in higher education. In: Huisman J (ed) International perspectives on the governance of higher education: alternative frameworks for coordination. Routledge, London, pp 1-13

13. Gupta AK, Smith KG, Shalley CE (2006) The interplay between exploration and exploitation. Acad Manag J 49(4):693-706

14. Cohen MD, March JG, Olsen JP (1972) A garbage can model of organizational choice. Adm Sci Q 17(1):1-23

15. Office of the Auditor General (2004) The office of the auditor general's investigation of the management of the universities. The Ministry of Education and Research, Oslo

16. Musselin C, Teixeira PN (2014) Reforming higher education: public policy design and implementation. Springer, Dordrecht

17. Huisman J, Stensaker B, Kehm BM (2009) Bologna, Quo Vadis? In: Kehm BM, Huisman J, Stensaker B (eds) The European Higher Education Area: Perspectives on a Moving Target. Sense Publishers, Rotterdam, pp xiii-xx

18. Jansen D, Pruisken (eds) (2015) The changing governance of higher education and research: multilevel perspectives. Springer, Dordrecht

19. Tucker CM (2003) The Lisbon Strategy and the Open Method of Coordination: A New Vision and the Revolutionary Potential of Soft Governance in the European Union. University of California Berkeley, Political Science Department. http://citeseerx.ist.psu. edu/viewdoc/download?doi=10.1.1.476.8411\&rep=rep1\&type= pdf. Accessed 31 August 2016
20. EHEA (2010) Communiqué of the 2010 Budapest-Vienna Ministerial Conference. http://media.ehea.info/file/2010 Budapest_Vienna/64/0/Budapest-Vienna_Declaration_598640. pdf. Accessed 31 August 2016

21. Huisman J, Westerheijden DF (2010) Bologna and quality assurance: progress made or pulling the wrong cart? Qual High Educ 16(1):63-66

22. EURASHE (2015) Standards and Guidelines for Quality Assurance in the European Higher Education Area (ESG). Brussels, Belgium

23. EHEA (2015) Communiqué of the 2015 Yerevan Ministerial Conference. http://media.ehea.info/file/2015 Yerevan/70 /7/YerevanCommuniqueFinal_613707.pdf. Accessed 31 August 2016

24. Romanelli E, Tushman ML (1994) Organizational transformation as punctuated equilibrium: an empirical test. Acad Manag J 37(5): $1141-1166$

25. Wischnevsky JD, Damanpour F (2005) Punctuated equilibrium model of organizational transformation: sources and consequences in the banking industry. Res Organ Chang Dev 15:207-239

26. European Commission/EACEA/Eurydice (2012) The European higher education area in 2012: Bologna process implementation report. Publications Office of the European Union, Luxembourg

27. EHEA (2012) Making the Most of Our Potential: Consolidating the European Higher Education Area. Communiqué of the 2012 Bucharest Ministerial Conference. http://media.ehea.info/file/2012 Bucharest/67/3/Bucharest_Communique_2012_610673.pdf. Accessed 31 August 2016

28. European Commission/EACEA/Eurydice (2015) The European higher education area in 2015: Bologna process implementation report. Publications Office of the European Union, Luxembourg

29. Gudmund Hernes (2013) Super-Resilient Organizations. In Karlsen JE, Pritchard RMO (eds) Resilient universities. Confronting changes in a challenging. World. Peter Lang, Bern, pp 381-402

30. Box GEP, Draper NR (1987) Empirical model building and response surfaces. John Wiley \& Sons, New York 